\title{
A multi-centered, retrospective, descriptive study on 107 dead patients with COVID-19
}

chong zhang

Wuhan University Zhongnan Hospital

Minhao Wu

Wuhan University Zhongnan Hospital

Xiaobin Zhu

Wuhan University Zhongnan Hospital

meng wu

Wuhan University Zhongnan Hospital

yufan zhu

Wuhan University Zhongnan Hospital

kun li

Wuhan University Zhongnan Hospital zhouming deng

Wuhan University Zhongnan Hospital

yuanlong xie

Wuhan University Zhongnan Hospital

lin cai ( $\nabla$ orthopedics@whu.edu.cn )

Wuhan University Zhongnan Hospital

Research article

Keywords: COVID-19, Mortality, SARS-CoV-2

Posted Date: April 8th, 2020

DOI: https://doi.org/10.21203/rs.3.rs-20836/v1

License: (c) (i) This work is licensed under a Creative Commons Attribution 4.0 International License.

Read Full License 


\section{Abstract}

Since the emergence of Corona Virus Disease 2019 (COVID-19) in Wuhan city, Hubei Province, China, it has caused thousands of deaths. As the ongoing outbreak of COVID-19 around the world, the number of deaths will definitely continue to increase. We aimed to further describe the clinical characteristics of dead cases with COVID-19 through a large sample and multi-centered study and to find some clinical predictors for the deterioration of COVID-19 during the process. Methods One hundred and seven patients (16 patients from Lei Shen-Shan Hospital, 54 patients from Seventh Hospital of Wuhan and 37 patients from Zhongnan Hospital of Wuhan University) with COVID-19 were enrolled in our research from Jan 22 to Feb 29, 2020. The demographic, clinical, radiological, laboratory and treatment data of all cases were analysed. Results Of the 107 dead patients with COVID-19, 71 (66.4\%) were male and 36 (33.6\%) were female. The mean age of the patients was $71.2 \pm 12.1$ years. $82(76.6 \%)$ of patients had chronic diseases. The mean duration from admission to death was 9 (IQR,5-14) days. Respiratory functional damage was the most common one followed by heart and kidney. Hematuria was found in 36(33.6\%) patients. 89(83.2\%) patients' albumin levels were decreased. 68(63.6\%) patients had anemia. concerning laboratory results, $55(69.6 \%)$ and $56(70.1 \%)$ patients have the elevated white blood cells and elevated Neutrophils during the process; only $43(54.4 \%)$ have the decreased Lymphocytes; The values of platelets and haemoglobin decreased in $64(81.0 \%)$ and 58 (73.4\%) patients. Alanine aminotransferase and aspartate aminotransferase elevated in near half of patients, while almost $80 \%$ of patients have the decreased albumin. The elevated blood urea nitrogen and cystatin $\mathrm{C}$ were manifested in about $70 \%$ of patients. Procalcitonin was elevated in $38(71.7 \%)$ patients. Conclusions In conclusion, the older men with chronic diseases are more likely to die from COVID-19. Apart from that, more attention should be pay on timely treatment, coinfections, malnutrition, and dysfunction of kidney and coagulation. The rising values (white blood cell, blood urea nitrogen, cystatin C, PCT and PT) and the decreased values (PLT, Hb and albumin) maybe meaningful for predict the poor prognosis.

\section{Background}

Since occurred in Wuhan (Hubei Province, China) in December 2019, severe acute respiratory syndrome coronavirus 2 (SARS-CoV-2) pneumonia has spread around the world becoming a public health emergency.[1-3] With rapidly increasing cases and local community transmission worldwide, the outbreak has entered a new phase.[4] As of Mar 11,2020, the total number of cases has increased to 118 322 in China and in other countries (including Thailand, Japan, South Korea, and the USA), with 4292 (3.6\%) deceased. Total number of deaths and requirement of intensive care will definitely continue to increase as the ongoing outbreak of COVID-19 around the world.[5] It has been reported that the mortality of critically ill patients reached to $61.5 \%$, and many factors, such as age, gender and chronic disease, have been thought to be associated with poor prognosis. [6-10] But the data on the clinical characteristics of multi-centered death patients with SARS-CoV-2 infection are rare, which are important to reduce mortality. 
In this study, we investigated 107 death cases with SARS-CoV-2 infection and analysed the changes of laboratory findings during the process. The clinical course data from this research will play an important role in early identifying the latent critically ill cases and providing more reasonable treatment for them.

\section{Methods}

\section{Study design and patients}

We performed this multi-centered, retrospective and descriptive research at Lei Shen-Shan Hospital (Center A), Seventh Hospital of Wuhan (Center B) and Zhongnan Hospital of Wuhan University (Center C). All of them are designated to treat patients with COVID-19 and managed by Zhongnan Hospital of Wuhan University. All dead patients, diagnosed with COVID-19 based on the WHO's interim guidelines, were included in our sturdy from Jan 22 to Feb 29, 2020. If patients were coinfected with any one of nine respiratory pathogens and the nucleic acid of influenza viruses $A$ and $B$, they were excluded. This study was reviewed and approved by the Medical Ethical Committee of ZhongNan Hospital of Wuhan University.

\section{Data Collection}

All data were collected from the information system of hospital, including demographic, clinical, radiological, laboratory and treatment data. All data were cross-checked. Missing and uncertain records were excluded if which can not be provided or clarified by involved health-care providers and their families.

\section{Statistical analysis}

Continuous variables were reported as mean \pm SD if they were normally distributed; otherwise, median (IQR) was used. Categorical variables were reported as number and percentage. Owing to different standards for the same laboratory test, we only assessed whether the measurements were outside the normal range and did not count the average values of the laboratory tests. The first laboratory findings after admission is defined as the first laboratory tests within 24 hours after admission to hospital; The last laboratory findings before death is also defined as the last laboratory tests within 24 hours before death; if the patients died within 24 hours after admission and some laboratory only be done once, the laboratory findings was treated as the first laboratory findings after admission.Data were analysed using the SPSS 21.0 statistical software (SPSS Inc., Chicago IL, USA).

\section{Results}

\section{Demographics and baseline characteristics}


107 patients ( 16 patients from Center A, 54 patients from Center $B$ and 37 patients from Center C) with COVID-19 were included in our research (Fig. 1), 71 (66.4\%) were male and 36 (33.6\%) were female. The mean age was $71.2 \pm 12.1$ years, and 62 (57.9\%) were older than 70 years. 82 (76.6\%) of those who died had chronic diseases, the most common of which was hypertension, followed by cardiovascular diseases, diabetes, chronic pulmonary disease kidney diseases, cerebrovascular diseases, malignant tumors, digestive system disease and chronic liver disease. (Table.1) The mean duration from onset of symptoms to admission was 7 (IQR,4-11) days. The mean duration from admission to death was 9 (IQR,5-14) days. The average course of the disease was 18 (IQR,13-24.5) days.

\section{Clinical Characteristics And Treatment Of Patients}

The most common symptoms were fever (88.2\%), cough (68.2\%), and dyspnoea (56.1\%). All patients had organ function damage.[11] Except respiratory function, the cardiac functional damage was the most common outside the lungs, followed by kidney and liver. $9(8.4 \%)$ patients had gastrointestinal haemorrhage. At the same time, Hematuria was found in $36(33.6 \%)$ patients, $4(3.7 \%)$ of them had urinary tract infection. Hospital-acquired pneumonia was found in $9(8.4 \%)$ patients, bacteraemia in $2(1.9 \%)$ patients. Only one patient with invasive mechanical ventilation had pneumothorax. Besides, 89(83.2\%) patients' albumin levels were decreased. 68(63.6\%) patients had anemia. (table 2)

As for treatment, all patients were treated in isolation. Antibacterial and antiviral treatment were given to 91 and 75 patients respectively, 51 patients received glucocorticoids. 53 patients were treated with highflow nasal cannula, 83 with mechanical ventilation, 5 patients with extracorporeal membrane oxygenation (ECMO), 4 with renal replacement therapy (table 2).

\section{Laboratory Results Of Patients With 2019-ncov Pneumonia On Admission}

After admission to hospital, leucocytes of 36 (39.6\%) patients and neutrophils of 46 (50.6\%) patients were above the normal range; leukopenia is present in 9 (9.9\%)patients; Lymphocytes and haemoglobin were below the normal range in $76(83.5 \%)$ and 56 (61.5\%) patients respectively; Platelets were below the normal range in $34(37.4 \%)$ patients and above the normal range in $3(3.3 \%) .35(51.5 \%)$ patients had abnormal myocardial zymogram as the elevation of any of creatine kinase MB form, lactate dehydrogenase and troponin. 68 (76.4\%) patients had abnormal liver function, with elevated alanine aminotransferase (ALT) and/or aspartate aminotransferase (AST). 64 (71.9\%) patients had different degrees of renal function damage, with elevated blood urea nitrogen or/and serum creatinine. As for the infection index, procalcitonin (PCT) was above the normal range in $67(91.8 \%)$ patients. Creactive protein (CRP) was above the normal range in $28(73.7 \%)$ patients (Table 3$)$. 
Table 1

Demographics and baseline characteristics of patients

\begin{tabular}{|ll|}
\hline & Patients $(\mathbf{n}=107)$ \\
\hline Gender & \\
\hline Male & $71(66.4 \%)$ \\
\hline Female & $36(33.6 \%)$ \\
\hline Age, years & \\
\hline Mean (SD) & $71.2(12.1)$ \\
\hline 50 & $5(4.7 \%)$ \\
\hline $61-70$ & $9(8.4 \%)$ \\
\hline $71-80$ & $31(29.0)$ \\
\hline $81-90$ & $37(34.6 \%)$ \\
\hline$\geq 91$ & $23(21.5 \%)$ \\
\hline Chronic diseases & $2(1.9 \%)$ \\
\hline Any & \\
\hline Hypertension & $82(76.6 \%)$ \\
\hline Cardiovascular diseases & $58(54.21 \%)$ \\
\hline Cerebrovascular diseases & $28(26.17 \%)$ \\
\hline Diabetes & $11(10.28 \%)$ \\
\hline Malignant tumors & $25(23.36 \%)$ \\
\hline Chronic pulmonary disease & $10(9.35 \%)$ \\
\hline Digestive system disease & $17(15.89 \%)$ \\
\hline Chronic liver disease & $9(8.41 \%)$ \\
\hline Chronic kidney diseases & $3(2.80 \%)$ \\
\hline Table.2 Symptoms, comorbidities, and treatments of patients \\
\hline
\end{tabular}


Table 2

Symptoms, comorbidities, and treatments of patients

\begin{tabular}{|ll|}
\hline & Patients $(\mathbf{n}=107)$ \\
\hline Symptoms & \\
\hline Fever & $88(88.2 \%)$ \\
\hline Cough & $73(68.2 \%)$ \\
\hline Sputum production & $52(48.6 \%)$ \\
\hline Dyspnoea & $60(56.1 \%)$ \\
\hline Fatigue & $55(51.4 \%)$ \\
\hline Poor appetite & $12(22.2 \%)$ \\
\hline Myalgia & $11(10.3 \%)$ \\
\hline Diarrhea & $8(7.5 \%)$ \\
\hline Chest pain & $3(2.8 \%)$ \\
\hline Coma & $1(0.9 \%)$ \\
\hline Vomiting & $1(0.9 \%)$ \\
\hline comorbidities & \\
\hline Acute respiratory distress syndrome & $92(86.0 \%)$ \\
\hline Acute kidney injury & $35(32.7 \%)$ \\
\hline Cardiac injury & $53(49.5 \%)$ \\
\hline Liver dysfunction & $26(24.3 \%)$ \\
\hline Anemia & $68(63.6 \%)$ \\
\hline Hypoalbuminemia & $89(83.2 \%)$ \\
\hline Hospital-acquired pneumonia & $9(8.4 \%)$ \\
\hline Bacteraemia & $2(1.9 \%)$ \\
\hline Gastrointestinal haemorrhage & $9(8.4 \%)$ \\
\hline Hematuria & $36(33.6 \%)$ \\
\hline Urinary tract infection & $4(3.7 \%)$ \\
\hline Pneumothorax & $1(0.9 \%)$ \\
\hline Treatments & \\
\hline
\end{tabular}




\begin{tabular}{|ll|}
\hline & Patients (n= 107) \\
\hline High flow nasal cannula & $53(49.5 \%)$ \\
\hline Mechanical ventilation & $93(86.9 \%)$ \\
\hline Non-invasive & $83(77.6 \%)$ \\
\hline Invasive & $66(61.7 \%)$ \\
\hline Antiviral agents & $83(77.6 \%)$ \\
\hline Antibacterial agents & $91(85.1 \%)$ \\
\hline Glucocorticoids & $58(54.2 \%)$ \\
\hline Renal replacement therapy & $4(3.7 \%)$ \\
\hline Extracorporeal membrane oxygenation & $5(4.7 \%)$ \\
\hline
\end{tabular}


Table 3

laboratory results of patients after admission

\begin{tabular}{|c|c|c|c|c|c|}
\hline laboratory findings & Center A & Center B & Center C & Total & percentage \\
\hline \multicolumn{6}{|l|}{ Routine blood test } \\
\hline \multicolumn{6}{|l|}{ White blood cells } \\
\hline Increased & $11(15)$ & $16(48)$ & $9(28)$ & $36(91)$ & $39.6 \%$ \\
\hline Decreased & $0(15)$ & $5(48)$ & $4(28)$ & $9(91)$ & $9.9 \%$ \\
\hline \multicolumn{6}{|l|}{ Neutrophils } \\
\hline Increased & $14(15)$ & $19(48)$ & $13(28)$ & $46(91)$ & $50.6 \%$ \\
\hline \multicolumn{6}{|l|}{ Lymphocytes } \\
\hline Decreased & $13(15)$ & $42(48)$ & $21(28)$ & $76(91)$ & $83.5 \%$ \\
\hline \multicolumn{6}{|l|}{ Platelets } \\
\hline Increased & $2(15)$ & $0(48)$ & $1(28)$ & $3(91)$ & $3.3 \%$ \\
\hline decreased & $7(15)$ & $15(48)$ & 12(28) & $34(91)$ & $37.4 \%$ \\
\hline \multicolumn{6}{|l|}{ Haemoglobin } \\
\hline decreased & $9(15)$ & $29(48)$ & $18(28)$ & $56(91)$ & $61.5 \%$ \\
\hline \multicolumn{6}{|l|}{ Cardiac biochemistry } \\
\hline Any & & & & $35(71)$ & $51.5 \%$ \\
\hline \multicolumn{6}{|l|}{ Myoglobin } \\
\hline Increased & $10(14)$ & $20(34)$ & 10(20) & $40(68)$ & $58.2 \%$ \\
\hline \multicolumn{6}{|l|}{ CKMB } \\
\hline Increased & $5(14)$ & $9(34)$ & $6(23)$ & $20(71)$ & $28.2 \%$ \\
\hline \multicolumn{6}{|c|}{ Lactate dehydrogenase } \\
\hline Increased & N/A & $\mathrm{N} / \mathrm{A}$ & 15(15) & $15(15)$ & $100 \%$ \\
\hline \multicolumn{6}{|l|}{ cTnl } \\
\hline Increased & $8(14)$ & N/A & $10(24)$ & $18(38)$ & $47.4 \%$ \\
\hline
\end{tabular}

CKMB: Creatine Kinase-MB Form. BNP: Brain natriuretic peptide. CTnl/T: cardiac troponin I/T; PT: Prothrombin time. APTT: Activated partial thromboplastin time. N/A: Not available.

Any: number of patients with any abnormal values 


\begin{tabular}{|c|c|c|c|c|c|}
\hline laboratory findings & Center A & Center B & Center C & Total & percentage \\
\hline Increased & N/A & $7(9)$ & N/A & $7(9)$ & $77.8 \%$ \\
\hline \multicolumn{6}{|l|}{ Pro-BNP } \\
\hline Increased & N/A & $3(8)$ & $3(6)$ & $6(14)$ & $42.9 \%$ \\
\hline \multicolumn{6}{|l|}{ BNP } \\
\hline Increased & 10(15) & 14(19) & $9(23)$ & $33(57)$ & $57.9 \%$ \\
\hline \multicolumn{6}{|l|}{ Liver biochemistry } \\
\hline Any & & & & $68(89)$ & $76.4 \%$ \\
\hline \multicolumn{6}{|c|}{ Alanine aminotransferase } \\
\hline Increased & $7(15)$ & $12(46)$ & $8(28)$ & 27(89) & $30.3 \%$ \\
\hline \multicolumn{6}{|c|}{ Aspartate aminotransferase } \\
\hline Increased & 11(15) & $33(46)$ & $24(28)$ & 68(89) & $76.4 \%$ \\
\hline \multicolumn{6}{|l|}{ Total bilirubin } \\
\hline Increased & $5(15)$ & $9(46)$ & $7(28)$ & $21(89)$ & $23.6 \%$ \\
\hline \multicolumn{6}{|l|}{ Albumin } \\
\hline Decreased & 15(15) & $44(46)$ & $27(28)$ & 86(89) & $96.6 \%$ \\
\hline \multicolumn{6}{|l|}{ Kidney biochemistry } \\
\hline Any & & & & 64(89) & $71.9 \%$ \\
\hline \multicolumn{6}{|l|}{ Blood urea nitrogen } \\
\hline Increased & $15(15)$ & $19(46)$ & $17(28)$ & $51(89)$ & $57.3 \%$ \\
\hline Decreased & $0(15)$ & $1(46)$ & $1(28)$ & $2(89)$ & $2.3 \%$ \\
\hline \multicolumn{6}{|l|}{ Serum creatinine } \\
\hline Increased & $9(15)$ & $18(46)$ & $12(28)$ & 39(89) & 43.8 \\
\hline \multicolumn{6}{|l|}{ Cystatin C } \\
\hline Increased & $12(15)$ & $22(27)$ & $24(27)$ & $58(69)$ & $84.1 \%$ \\
\hline
\end{tabular}

CKMB: Creatine Kinase-MB Form. BNP: Brain natriuretic peptide. cTnl/T: cardiac troponin I/T; PT: Prothrombin time. APTT: Activated partial thromboplastin time. N/A: Not available.

Any: number of patients with any abnormal values 


\begin{tabular}{|c|c|c|c|c|c|}
\hline laboratory findings & Center A & Center B & Center C & Total & percentage \\
\hline \multicolumn{6}{|l|}{ C-reactive protein } \\
\hline Increased & N/A & $21(21)$ & $7(17)$ & $28(38)$ & $73.7 \%$ \\
\hline \multicolumn{6}{|l|}{ Procalcitonin } \\
\hline Increased & 14(14) & 28(34) & $25(25)$ & 67(73) & $91.8 \%$ \\
\hline \multicolumn{6}{|l|}{ Coagulation function } \\
\hline \multicolumn{6}{|l|}{ PT } \\
\hline Increased & $4(15)$ & $17(27)$ & $15(26)$ & $36(68)$ & $52.9 \%$ \\
\hline Decreased & $1(15)$ & $0(27)$ & $0(26)$ & $1(68)$ & $1.5 \%$ \\
\hline \multicolumn{6}{|l|}{ APTT } \\
\hline Increased & $3(15)$ & $7(36)$ & $9(26)$ & 19(77) & $24.7 \%$ \\
\hline Decreased & $0(15)$ & $1(36)$ & $1(26)$ & $2(77)$ & $2.6 \%$ \\
\hline \multicolumn{6}{|l|}{ D-dimer } \\
\hline Increased & $15(15)$ & $20(20)$ & $14(25)$ & $49(60)$ & $81.7 \%$ \\
\hline \multicolumn{6}{|c|}{$\begin{array}{l}\text { CKMB: Creatine Kinase-MB Form. BNP: Brain natriuretic peptide. cTnl/T: cardiac troponin I/T; PT: } \\
\text { Prothrombin time. APTT: Activated partial thromboplastin time. N/A: Not available. }\end{array}$} \\
\hline Any: number of pati & bnormal va & & & & \\
\hline
\end{tabular}


Table 4

the changes of repeated laboratory results between the last and the first test

\begin{tabular}{|c|c|c|c|c|c|}
\hline laboratory findings & Center A & Center B & Center C & Total & percentage \\
\hline \multicolumn{6}{|l|}{ Routine blood test } \\
\hline White blood cells * & $6(13)$ & $31(41)$ & 18(25) & $55(79)$ & $69.6 \%$ \\
\hline Neutrophils & $7(13)$ & $30(41)$ & $19(25)$ & $56(79)$ & $70.1 \%$ \\
\hline Lymphocytes & 10(13) & $14(41)$ & 19(25) & $43(79)$ & $54.4 \%$ \\
\hline Platelets ** & $9(13)$ & $35(41)$ & $20(25)$ & 64(79) & $81.0 \%$ \\
\hline Haemoglobin & 12(13) & $29(41)$ & $17(25)$ & $58(79)$ & $73.4 \%$ \\
\hline \multicolumn{6}{|l|}{ Liver biochemistry } \\
\hline ALT & $3(12)$ & 18(37) & $11(21)$ & $32(70)$ & $45.7 \%$ \\
\hline AST & $5(12)$ & $16(37)$ & $10(21)$ & $31(70)$ & $44.3 \%$ \\
\hline Total bilirubin & $8(12)$ & 18(37) & $15(21)$ & $41(70)$ & $58.6 \%$ \\
\hline Albumin & $9(12)$ & $27(37)$ & $19(21)$ & $55(70)$ & $78.6 \%$ \\
\hline \multicolumn{6}{|l|}{ Kidney biochemistry } \\
\hline Blood urea nitrogen* & $7(14)$ & 26(39) & 19(20) & $52(73)$ & $71.2 \%$ \\
\hline Serum creatinine & $7(14)$ & 20(39) & 16(20) & $43(73)$ & $58.9 \%$ \\
\hline cystatin C & $8(14)$ & $6(11)$ & $17(20)$ & $31(45)$ & $68.9 \%$ \\
\hline \multicolumn{6}{|c|}{ Inflammation indicators } \\
\hline C-reactive protein & N/A & $4(6)$ & $2(4)$ & $6(10)$ & $60.0 \%$ \\
\hline Procalcitonin & $8(13)$ & $13(21)$ & 17(19) & $38(53)$ & $71.7 \%$ \\
\hline \multicolumn{6}{|l|}{ Coagulation function } \\
\hline $\mathrm{PT}$ * & $9(14)$ & 19(24) & $14(23)$ & $42(61)$ & $68.9 \%$ \\
\hline APTT * & $9(14)$ & $14(28)$ & 15(23) & $38(65)$ & $58.5 \%$ \\
\hline D-dimer & $8(14)$ & $5(10)$ & 12(19) & $25(43)$ & $58.1 \%$ \\
\hline
\end{tabular}

PT: Prothrombin time. APTT: Activated partial thromboplastin time. CKMB: Creatine Kinase-MB Form. BNP: Brain natriuretic peptide; CTnl/T :cardiac troponin I/T; N/A: Not available.

*: the increase of these values is analysesd, not decrease has been found. **: the decrease of this value is analysesd, not increase has been found. 


\begin{tabular}{|llllll|}
\hline laboratory findings & Center A & Center B & Center C & Total & percentage \\
\hline Myoglobin & $6(7)$ & $9(16)$ & $10(12)$ & $25(35)$ & $71.4 \%$ \\
\hline CK-MB & $10(11)$ & $8(15)$ & $5(5)$ & $23(30)$ & $74.2 \%$ \\
\hline Lactate dehydrogenase & N/A & N/A & $3(3)$ & $3(3)$ & $100 \%$ \\
\hline Troponin I & $4(8)$ & N/A & $10(15)$ & $14(23)$ & $60.9 \%$ \\
\hline Troponin T & N/A & $3(3)$ & N/A & $3(3)$ & $100 \%$ \\
\hline Pro-BNP & N/A & $4(7)$ & $2(2)$ & $6(9)$ & $66.7 \%$ \\
\hline BNP & $7(13)$ & $8(11)$ & $7(10)$ & $22(34)$ & $64.7 \%$ \\
\hline $\begin{array}{l}\text { PT: Prothrombin time. APTT: Activated partial thromboplastin time. CKMB: Creatine Kinase-MB Form. } \\
\text { BNP: Brain natriuretic peptide; CTnl/T :cardiac troponin I/T; N/A: Not available. }\end{array}$ & $\begin{array}{l}\text { *: the increase of these values is analysesd, not decrease has been found. **: the decrease of this } \\
\text { value is analysesd, not increase has been found. }\end{array}$ \\
\hline
\end{tabular}

\section{The changes of repeated measurements between last one before death and the first after admission}

Comparing the repeated value of the last and the first test, we find that $55(69.6 \%)$ patients have the elevated white blood cells; 56 (70.1\%) patients have the elevated Neutrophils; only $43(54.4 \%)$ have the decreased Lymphocytes; The values of platelets and haemoglobin are decreased in 64(81.0\%) and 58 (73.4\%)patients. As for liver function, elevated alanine aminotransferase and aspartate aminotransferase are found in near half of patients, while almost $80 \%$ of patients have the decreased albumin. Concerning the renal function, the elevated blood urea nitrogen and cystatin $C$ were manifested in about $70 \%$ of patients. Procalcitonin was elevated in 38 (71.7\%) patients. Prothrombin time is longer in $42(68.9 \%)$ patients. The elevations of myoglobin, Creatine Kinase-MB Form, Pro-Brain natriuretic peptide, Brain natriuretic peptide and Troponin I is found in about $70 \%$ of patients, the elevation of lactate dehydrogenase and troponin $\mathrm{T}$ were found more than $90 \%$ of patients.

\section{Discussion}

In our research, 107 death patients confirmed with COVID-19 had been included. The average age of all dead patients is $71.2 \pm 12.1$ years, male accounted for $66.4 \%$. As it had been reported that the older and male patients are more likely to be infected with SARS-CoV-2,[12] and the male and older patients with viral pneumonia are also prone to develop ARDS,[13] which is the main cause of death in our research. Some research found that $\mathrm{X}$ chromosome and sex hormones could protect females from viral infections. [14] All above may explain why age and gender were associated with death.

Chronic diseases had been treated as important risk factors for poor outcome of COVID-19 as well.[15] In a research about critically ill patients with SARS-CoV-2 pneumonia, $50 \%$ of non-survivors have chronic diseases, while $25 \%$ for survivors.[8] As for our research, $76.6 \%$ of dead cases had chronic underlying 
diseases, the most common one was hypertension, followed by cardiovascular diseases. At the same time, cardiac injuries were most common cause of death behind respiratory failure (Fig. 2), followed by the injury of kidney and liver. As mentioned above, we guess that SARS-CoV-2 may worsen the injury of heart, exacerbating the death of patients. So, for critically ill patients, more attention should be focus on the injury of heart.

Most patients with SARS-CoV-2 infection had fever, $[8,12]$ which is in accordance with our studies. But research found that $11.5 \%$ of patients did not have fever at the onset of illness and thought this make the early identification more difficult.[8] There are also 11(12.5\%) out of 88 patients with fever in our research who did not manifest fever at the onset of symptoms. At the same time, the median duration from onset of symptoms to ICU admission was 5 (3-7) days,[8] while the median duration from onset of symptoms to admission to hospital in our study is 7 (IQR,4-11) days. So the delay manifestation of fever and delay reception of medical care may exacerbate the death of patients with SARS-CoV-2 pneumonia.

It has been reported that the mortality rate in critically ill patients with SARS-CoV-2 infection can reach to $61.5 \%$, higher than that of SARS-CoV and Middle Eastern respiratory syndrome (MERS)-CoV.[8] Although many drugs are under clinical trial, there is no specific treatment for coronavirus infection at this time apart from supportive care.[13] 93 (86.9\%) patients in our research received mechanical ventilation, except for some patients whose relatives refused mechanical ventilation. $83(77.6 \%)$ dead cases were given oseltamivir or others and almost half of patients received methylprednisolone. Although the use of glucocorticoids for patients with COVID-19 is controversial[16] and no antiviral agents treating SARS and MERS have been found effective for SARS-CoV-2,[15] more researches are need.

As for laboratory findings, lymphocytopenia is a common feature of patients with SARS-CoV-2 infection. Similar to those with SARS-CoV and MERS infection, targeted invasion by the virus may results in the apoptosis of lymphocytes in patients with SARS-CoV-2 infection. $[17,18]$ In one research, $35 \%$ of noncritical patients with SARS-CoV-2 infection had mild lymphocytopenia.[12] While another research found that more than $80 \%$ of critically ill patients had lymphocytopenia and thought that the severity of lymphocytopenia reflects the severity of SARS-CoV-2 infection.[8] In our study, 83.5\% of dead patients in our study have lymphocytopenia on admission. Comparing with first value after admission, the absolute value of lymphocytes before death were decreased in 43 (54.4\%) of patients. Lymphocytopenia is good to diagnosis of SARS-CoV-2 infection, but whether the severity of lymphocytopenia can predict the prognosis of SARS-CoV-2 infection is unclear. It has been reported the presence of secondary infection and elevated inflammatory indicators is predictor of poor prognosis in COVID-19 by comparing laboratory parameters between survivors and non-survivors.[7] By comparing with first value after admission, the absolute value of white blood cells and Neutrophils before death were elevated in $55(69.6 \%)$ and 56 $(70.1 \%)$ of patients during the process of the disease. At the same time, PCT, an effective indicator of bacterial infection,[19] was elevated in $67(91.8 \%)$ patients on admission and 38 (71.7\%) of patients experienced the elevation of PCT before death. Besides, 9 patients with hospital-acquired pneumonia, 4 with urinary tract infection and 2 with bacteraemia had been found. The results of our research furtherly suggest that coinfections and inflammation do exacerbate the death of patients. 
As for organ dysfunction, cardiac damage is the most common one except for lung. Fulminant myocarditis is related to the rapid progress and a critically ill state of illness.[20] Concerning the laboratory parameters of heart, cardiac troponin and myoglobin of non-survivors is higher than survivors. [7] In our study, the elevations of myoglobin, CKMB, Pro-BNP, BNP and Troponin I is found in about $70 \%$ of patients, the elevation of lactate dehydrogenase and troponin T were found more than $90 \%$ of patients, during the process of disease. Myoglobin, Creatine Kinase-MB Form, BNP may predict the prognosis. But the other biomarker of cardiac function has been repeated in few patients, more researches are needed. On the other aspects, 68 (76.4\%) and 64 (71.9\%) patients had abnormal liver function and renal function damage on admission, which is higher than that of previous study.[21] we also found near $50 \%$ of patients experienced the elevation of alanine aminotransferase and aspartate aminotransferase. while the rate of elevated blood urea nitrogen $(71.2 \%)$ and cystatin $C(68.8 \%)$ is higher than Serum creatinine (58.9\%). The damage of kidney and liver maybe sever on the admission and during the process of COVID19. Blood urea nitrogen and cystatin $\mathrm{C}$ maybe sensitive to predict the change of renal function

In addition, 56 (61.5\%) patients' Hb levels and 34 (37.4\%) patients' PLT levels were below the normal range after admission. Comparing with the first one, 58 (73.4\%) patients' $\mathrm{Hb}$ and 64 (81.0\%) patients' PLT levels of last test before death were decreased. In terms of coagulation function, the first levels of PT increased in $36(52.9 \%)$ patients, the levels of APTT increased in $19(24.7 \%)$ patients. As for change of coagulation function, longer PT was found in 42 (68.9\%) patients, longer APTT was found in 38 (58.5\%) patients. At the same time, 36 patients have hematuria and 9 patients had gastrointestinal haemorrhage. We also should pay more attention to the change of coagulation function of critically ill patients. But

whether hematuria and gastrointestinal haemorrhage are associated with change of coagulation function is unclear. At last, 86 (96.6\%) patients' albumin levels were below the normal range at first test. What is more, the last level of albumin is decreased in 55 (78.6\%) patients, comparing with the first test. it indicates that most of severe patients may have malnutrition.

This study has several limitations. First, only dead patients with confirmed SARS-CoV-2 infection were included. It would be better to have discharged patients included in our research and compare the difference between them. Second, some dead patients did not have the repeated measurements of some laboratory findings, so the sample size of some part of this research is small. Third, some important laboratory results were not tested by the same way in three hospital, which may impact the research negatively. Finally, this is a retrospective study. The data in this study permit a preliminary assessment of the clinical course of critically ill patients with SARS-CoV-2 pneumonia. More researches are still needed.

\section{Conclusion}

In conclusion, the older men with chronic diseases are more likely to die from COVID-19. Apart from that, more attention should be pay on bacterial infections, malnutrition, and dysfunction of kidney and coagulation during the process of COVID-19. The rising of white blood cell, PCT, blood urea nitrogen, cystatin $\mathrm{C}$ and $\mathrm{PT}$ and decreased values of PLT, $\mathrm{Hb}$ and albumin may be meaningful for prediction the poor prognosis. 


\section{Abbreviations}

COVID-19: Corona Virus Disease 2019

SARS-CoV-2: severe acute respiratory syndrome coronavirus 2

ALT: alanine aminotransferase

AST: aspartate aminotransferase

PCT: procalcitonin

CRP: Creactive protein

CKMB: Creatine Kinase-MB form.

BNP: Brain natriuretic peptide.

cTnl/T :cardiac troponin I/T;

PT: Prothrombin time.

APTT: Activated partial thromboplastin time.

N/A: Not available

\section{Declarations}

\section{Acknowledgements}

Thanks to Dr. Zhiyong Peng and colleagues in Zhongnan Hospital of Wuhan University for the treatment of critically ill COVID-19 patients.

\section{Funding}

None

\section{Ethics approval and consent to participate}

This study was reviewed and approved by the Medical Ethical Committee of ZhongNan Hospital of Wuhan University (2020067). Informed consent waiver was obtained. To collect the data from the patients' record, permission was obtained from Medical Ethical Committee and Hospital Medical Department. All data used in research was anonymized before use.

\section{Patient data}


All data in our research has not been reported in any other submission by the time of submission to BMC infectious diseases.

\section{Consent for publication}

Not applicable

\section{Competing interests}

All authors declare that they have no competing interests.

\section{Contributions}

Lin Cai and Yuanlong Xie had full access to all of the data in the study and take responsibility for the integrity of the data and the accuracy of the data analysis. Chong Zhang, Minhao Wu and Xiaobin Zhu contributed equally and share first authorship. Lin Cai and Yuanlong Xie contributed equally to this article.

Concept and design: Lin Cai, Yuanlong Xie

Data collection: Chong Zhang, Minhao Wu, Xiaobin Zhu Yufan Zhu

Data analysis: Chong Zhang, Yufan Zhu, Meng Wu

Drafting of the manuscript: Chong Zhang, Minhao Wu, Xiaobin Zhu

Administrative, technical, or material support: Meng Wu, Kun Li, Zhouming Deng

All authors read and approved the final manuscript.

\section{References}

1. Lupia T, Scabini S, Pinna SM, Di Perri G, De Rosa FG, Corcione S: 2019-novel coronavirus outbreak: A new challenge. Journal of global antimicrobial resistance 2020.

2. Johnson HC, Gossner CM, Colzani E, Kinsman J, Alexakis L, Beaute J, Wurz A, Tsolova S, Bundle N, Ekdahl K: Potential scenarios for the progression of a COVID-19 epidemic in the European Union and the European Economic Area, March 2020. Euro surveillance: bulletin Europeen sur les maladies transmissibles = European communicable disease bulletin 2020, 25(9).

3. Lai CC, Shih TP, Ko WC, Tang HJ, Hsueh PR. Severe acute respiratory syndrome coronavirus 2 (SARSCoV-2) and coronavirus disease-2019 (COVID-19): The epidemic and the challenges. International journal of antimicrobial agents 2020:105924.

4. Maxwell DN, Perl TM, Cutrell JB: "The Art of War" in the Era of Coronavirus Disease 2019 (COVID-19). Clinical infectious diseases: an official publication of the Infectious Diseases Society of America 2020. 
5. Murthy S, Gomersall CD, Fowler RA: Care for Critically III Patients With COVID-19. Jama 2020.

6. Applegate WB, Ouslander JG. COVID-19 Presents High Risk to Older Persons. Journal of the American Geriatrics Society 2020.

7. Ruan Q, Yang K, Wang W, Jiang L, Song J. Clinical predictors of mortality due to COVID-19 based on an analysis of data of $\mathbf{1 5 0}$ patients from Wuhan, China. Intensive care medicine 2020.

8. Yang X, Yu Y, Xu J, Shu H, Xia Ja, Liu H, Wu Y, Zhang L, Yu Z, Fang M, et al: Clinical course and outcomes of critically ill patients with SARS-CoV-2 pneumonia in Wuhan, China: a single-centered, retrospective, observational study. Lancet Respir Med 2020:S2213-2600(2220)30079 - 30075..

9. Huang C, Wang Y, Li X, Ren L, Zhao J, Hu Y, Zhang L, Fan G, Xu J, Gu X, et al. Clinical features of patients infected with 2019 novel coronavirus in Wuhan, China. Lancet. 2020;395(10223):497-506.

10. Li K, Wu J, Wu F, Guo D, Chen L, Fang Z, Li C: The Clinical and Chest CT Features Associated with Severe and Critical COVID-19 Pneumonia. Investigative radiology 2020.

11. Cascella M, Rajnik M, Cuomo A, Dulebohn SC, Di Napoli R: Features, Evaluation and Treatment Coronavirus (COVID-19). In: StatPearls. Treasure Island (FL): StatPearls Publishing StatPearls Publishing LLC.; 2020.

12. Chen N, Zhou M, Dong X, Qu J, Gong F, Han Y, Qiu Y, Wang J, Liu Y, Wei Y, et al. Epidemiological and clinical characteristics of 99 cases of 2019 novel coronavirus pneumonia in Wuhan, China: a descriptive study. Lancet. 2020;395(10223):507-13.

13. de Wit E, van Doremalen N, Falzarano D, Munster VJ. SARS and MERS: recent insights into emerging coronaviruses. Nature reviews Microbiology. 2016;14(8):523-34.

14. Jaillon S, Berthenet K, Garlanda C. Sexual Dimorphism in Innate Immunity. Clin Rev Allergy Immunol. 2019;56(3):308-21.

15. Wang D, Hu B, Hu C, Zhu F, Liu X, Zhang J, Wang B, Xiang H, Cheng Z, Xiong Y, et al: Clinical Characteristics of 138 Hospitalized Patients With 2019 Novel Coronavirus-Infected Pneumonia in Wuhan, China. Jama 2020.

16. Russell CD, Millar JE, Baillie JK. Clinical evidence does not support corticosteroid treatment for 2019nCoV lung injury. Lancet. 2020;395(10223):473-5.

17. Gu J, Gong E, Zhang B, Zheng J, Gao Z, Zhong Y, Zou W, Zhan J, Wang S, Xie Z, et al. Multiple organ infection and the pathogenesis of SARS. The Journal of experimental medicine. 2005;202(3):41524.

18. Liu WJ, Zhao M, Liu K, Xu K, Wong G, Tan W, Gao GF. T-cell immunity of SARS-CoV: Implications for vaccine development against MERS-CoV. Antiviral research. 2017;137:82-92.

19. Schuetz P, Wirz Y, Sager R, Christ-Crain M, Stolz D, Tamm M, Bouadma L, Luyt CE, Wolff M, Chastre J, et al. Procalcitonin to initiate or discontinue antibiotics in acute respiratory tract infections. Cochrane Database Syst Rev. 2017;10:Cd007498.

20. Wang D, Li S, Jiang J, Yan J, Zhao C, Wang Y, Ma Y, Zeng H, Guo X, Wang H, et al. Chinese society of cardiology expert consensus statement on the diagnosis and treatment of adult fulminant 
myocarditis. Science China Life sciences. 2019;62(2):187-202.

21. Guan WJ, Ni ZY, Hu Y, Liang WH, Ou CQ, He JX, Liu L, Shan H, Lei CL, Hui DSC, et al: Clinical

Characteristics of Coronavirus Disease 2019 in China. The New England journal of medicine 2020.

\section{Figures}

16 dead patients from Center A

64 dead patients from Center B

42 dead patients from Center $\mathrm{C}$

Total 122 dead patients COVID-19 from Jan 22, 2020 to Feb 29, 2020

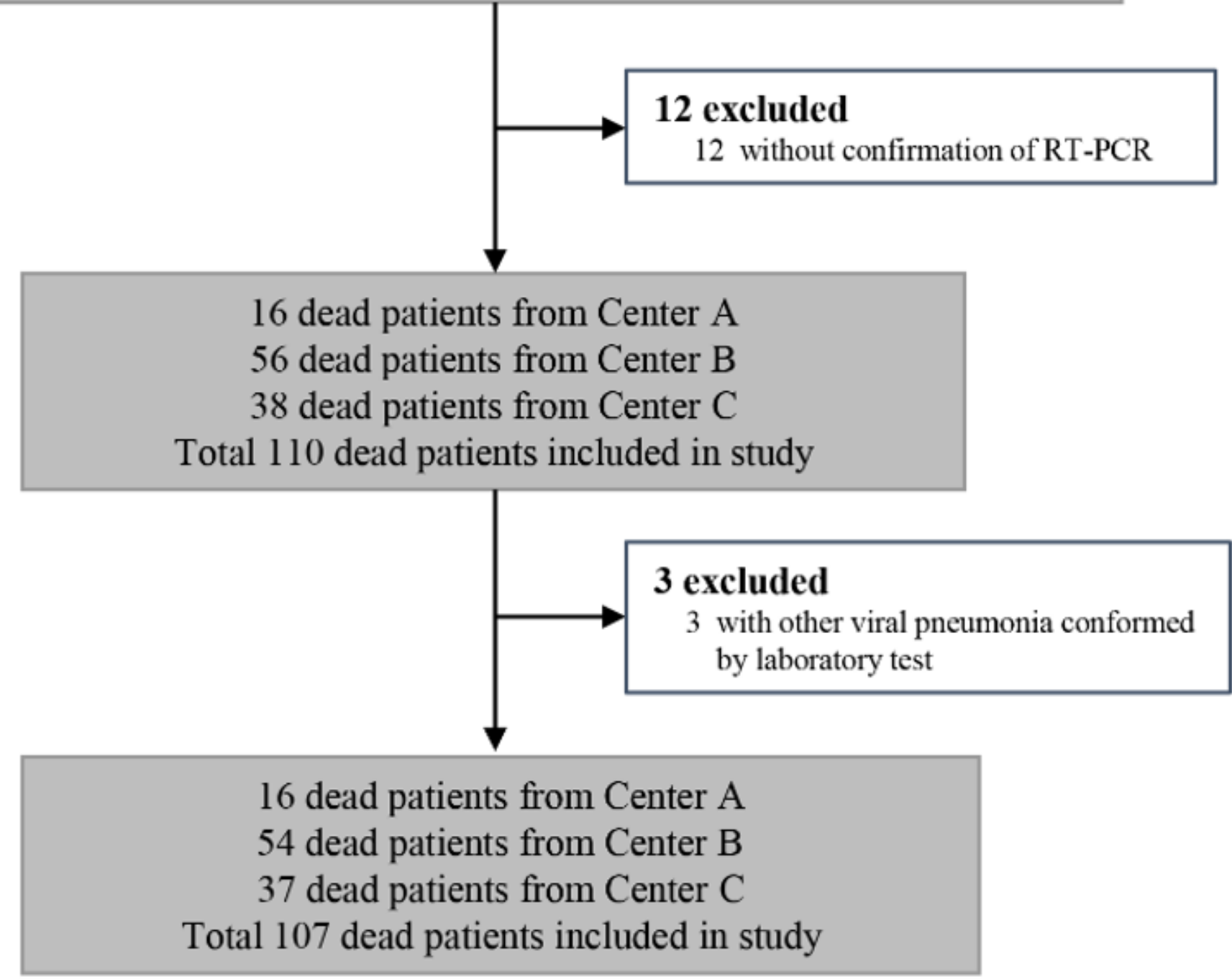

Figure 1

Study flow diagram 


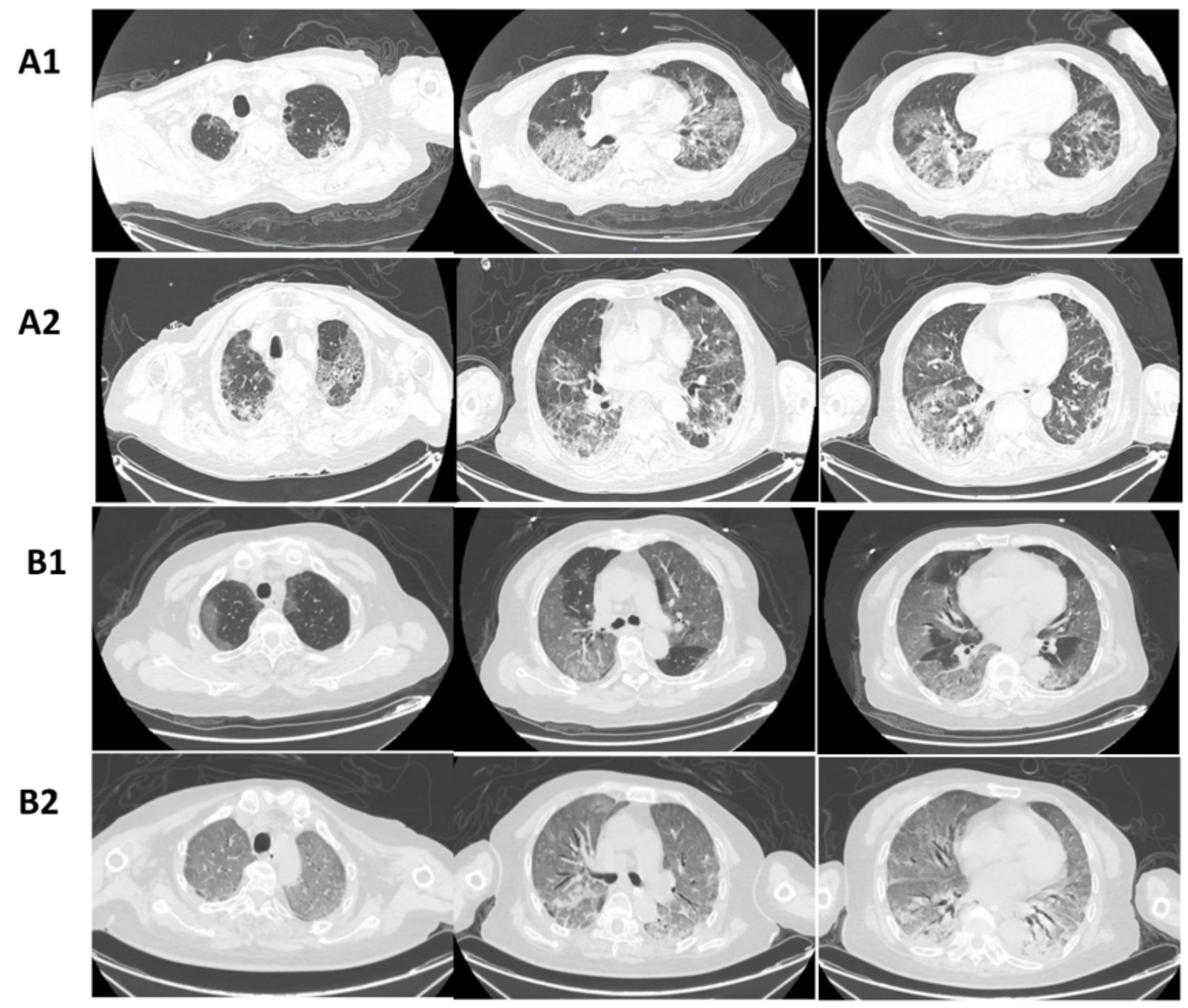

Figure 2

A1: the early stage Chest CT scan of typical case 1; A2: the late stage Chest CT scan of typical case 1 . B1: the early stage Chest CT scan of typical case 2; B2: the late stage Chest CT scan of P typical case 2. 